\title{
Originales
}

\section{Comparación de dos posiciones de electrodos en la cardioversión eléctrica de la fibrilación auricular}

\author{
T. MUÑOZ MARTÍNEZ, S. MARTÍNEZ ALÚTIZ, C. VINUESA LOZANO, Y. POVEDA HERNÁNDEZ, \\ J. L. DUDAGOITIA OTAOLEA, S. IRIBARREN DIARASARRI Y M. HERNÁNDEZ LÓPEZ
}

Unidad de Cuidados Intensivos. Hospital Txagorritxu. Vitoria-Gasteiz. España.

Objetivo. El objetivo de este estudio es comparar la efectividad de la cardioversión eléctrica (CVE) en pacientes con fibrilación auricular (FA) crónica según la posición de los electrodos: anteroapical (A-A) o anteroposterior (A-P). Se considera superior la que restaura el ritmo sinusal (RS) empleando menor energía.

Diseño. Estudio observacional comparando dos series consecutivas de pacientes.

Ámbito. Unidad de Cuidados Intensivos (UCI) de un hospital de segundo nivel.

Pacientes y métodos. Pacientes ambulatorios en $\mathrm{FA}$ remitidos a la $\mathrm{UCl}$ para CVE bifásica. En la primera serie se comenzaba con la posición A-A, y en la segunda con la A-P, administrándose hasta 3 choques (150-200-200J); de no haberse conseguido RS, se cambiaba a la posición alternativa y se administraban otros 2 choques más de 200J. Se analizaron y compararon entre ambas series la edad, sexo, peso, cardiopatía de base, fracción de eyección, tamaño auricular izquierdo, tiempo en FA, constantes basales, medicación antiarrítmica, reversión a RS, número de choques, energía empleada y efectos secundarios.

Resultados. Se trataron 50 pacientes en cada grupo, cuyas características basales eran similares salvo un porcentaje superior de mujeres en el grupo A-A. La posición de electrodos A-A consiguió RS con significativamente menor número de choques y menor energía, lográndose con más frecuencia la reversión al primer choque.

Correspondencia: Dr. T. Muñoz.

UCI. Hospital Txagorritxu.

C/ J. Achótegui, s/n

01009 Vitoria-Gasteiz. España.

Correo electrónico: tmunoz@htxa.osakidetza.net

Manuscrito aceptado el 21-XI-2005
Conclusiones. Encontramos mayor efectividad en la CVE de la FA con los electrodos en posición A-A, que recomendamos como primera elección. De no ser eficaz, debe intentarse la posición A-P.

PALABRAS CLAVE: fibrilación auricular, cardioversión eléctrica, choque bifásico, posición de electrodos.

\section{COMPARISON OF TWO ELECTRODE POSITIONS IN ELECTRICAL CARDIOVERSION OF ATRIAL FIBRILLATION}

Objective. This study aims to compare effectiveness of electrical cardioversion (ECV) in patients with chronic atrial fibrillation (AF) according to the electrode positions: anteroapical (A-A) or anteroposterior (A-P). That which restores the sinus rhythm (SR) using the least energy is considered superior.

Design. Observational study comparing two consecutive series of patients.

Scope. Intensive Care Unit (ICU) of second level hospital.

Patients and methods. Out-patients in AF referred to the ICU for biphasic ECV. The first series began with position A-A and the second one with $A-P$, administering up to 3 shocks (150-200200J), changing to the alternative position if SR was not achieved and administering 2 more shocks of 200J. Age, gender, weight, baseline heart disease, ejection fraction, left atrial size, AF time, baseline vital signs, antiarrhythmic medication, reversion to SR, number of shocks, energy used and side effects were analyzed and compared between both series.

Results. A total of $\mathbf{5 0}$ patients were treated in each group. The baseline characteristics were similar except for a greater percentage of women in group A-A. The anteroapical electrode position achieved SR with significantly fewer 
numbers of shocks and less energy, more frequently achieving reversion on the first shock. Conclusions. We found greater effectiveness in the electrical cardioversion of the AF with the electrodes in the anteroapical position, that we recommend as first choice. If it is not effective, the A-P position should be attempted.

KEY WORDS: atrial fibrillation, electrical cardioversion, biphasic shock, electrode position.

\section{INTRODUCCIÓN}

La cardioversión eléctrica (CVE) es una estrategia clave en el manejo de la fibrilación auricular (FA) como queda bien reflejado en las guías de diversas sociedades científicas ${ }^{1-3}$. Por este motivo es fundamental hacer dicho procedimiento lo más seguro y eficaz posible.

Según el tipo de paciente, puede ser preferible optar por el control de ritmo (restaurar ritmo sinusal mediante cardioversiones repetidas si es preciso y utilizar fármacos antiarrítmicos para evitar recidivas) o por el control de frecuencia (aceptar la FA y pautar fármacos bradicardizantes y anticoagulantes para evitar las complicaciones). Estas dos estrategias claves en el manejo de esta arritmia están razonadas en las guías ${ }^{2}$ y no se insistirá sobre ello, ya que nuestro trabajo se centra en la CVE.

Aunque se han publicado diversas recomendaciones para cardiovertir la $\mathrm{FA}^{4.6}$, los últimos trabajos recomiendan utilizar choques bifásicos y colocar los electrodos en posición anteroposterior $(\mathrm{A}-\mathrm{P})^{7}$, tal como comentan las guías más importantes ${ }^{2}$. Siguiendo estas recomendaciones, nuestro grupo, que inicialmente utilizaba la posición anteroapical (A-A) como primera opción en los pacientes en FA, cambió su protocolo a la A-P, manteniendo el resto del tratamiento sin modificaciones. Hemos registrado de forma prospectiva los datos de cada procedimiento, práctica que ya en otras ocasiones nos ha permitido analizar el impacto de cada cambio en nuestro proto$\mathrm{colo}^{8.9}$. El presente estudio analiza en nuestro medio la efectividad de la posición A-A frente a la A-P para restaurar el ritmo sinusal (RS) mediante choques bifásicos (onda bifásica exponencial truncada) en pacientes con FA crónica.

\section{PACIENTES Y MÉTODOS}

Se trata de un estudio observacional que compara dos series consecutivas de pacientes tratados en nuestra Unidad de Cuidados Intensivos (UCI) mediante cardioversión eléctrica con onda bifásica exponencial truncada.

\section{Población del estudio}

Son pacientes con FA crónica estable, correctamente anticoagulados (INR $>2$ en las 4 semanas

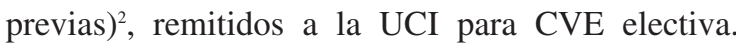
Todos habían firmado el correspondiente consentimiento para la técnica y específicamente para incluir sus datos en el registro. Algunos pacientes llevaban medicación antiarrítmica de base, según preferencias de su cardiólogo referente, frecuentemente amiodarona con el fin de evitar recurrencias.

\section{Protocolo de CVE}

En ayunas desde la noche anterior, los pacientes ingresaban en la Unidad de Día, donde tras comprobar persistencia de la arritmia, canalizar vía venosa periférica y confirmar parámetros analíticos adecuados, eran trasladados a la UCI para realizar el procedimiento. Se monitorizaban el ritmo cardiaco (tres derivaciones), la presión arterial de manera no invasora y la pulsioximetría. Todos los pacientes recibían oxígeno al 50\% mediante mascarilla. Tras inducción con propofol ( $1 \mathrm{mg} / \mathrm{kg}$ en 1 minuto) y remifentanilo $(0,5 \mu \mathrm{g} / \mathrm{kg}$ en el siguiente minuto), se administraban a través de electrodos adhesivos choques eléctricos bifásicos sincronizados, con un desfibrilador Agilent Smart Biphasic.

En la primera serie de pacientes la posición inicial de los electrodos era la A-A y en la segunda la A-P. Se administraban hasta 3 choques $(150,200$, 200 J) en esa posición y, de no obtenerse RS, se cambiaba a la posición alternativa administrándose hasta 2 choques más con energía máxima (200 J).

Tras los choques, se mantenía a los pacientes en la UCI hasta 15 minutos tras recuperar un nivel de conciencia normal. De no aparecer ningún problema, se trasladaban de nuevo a la Unidad de Día, donde recibían analgesia (paracetamol por vía oral $1 \mathrm{~g}$ ) y cuidados en la superficie cutánea expuesta a la corriente eléctrica (pomada de sulfadiazina). Dos horas más tarde, comprobada la estabilidad y la tolerancia a la dieta, eran dados de alta a su domicilio.

\section{Variables analizadas}

Se analizaron edad, sexo, peso, cardiopatía de base, fracción de eyección (FE) ventricular izquierda, tamaño de aurícula izquierda (AI), tiempo de evolución de la FA, medicación antiarrítmica coadyuvante, presión arterial media y frecuencia cardíaca (FC) basales, reversión a RS, número de choques, energía empleada (en los casos revertidos), necesidad de cambio de posición de electrodos para conseguir RS, soporte hemodinámico (definido como necesidad de actuación sobre la FC o la presión arterial), soporte respiratorio (necesidad de ventilación con bolsa-mascarilla) y efectos secundarios.

\section{Estadística}

Se compararon ambos grupos mediante los métodos $\chi^{2}$ (para variables categóricas) y «t» de Student (para las continuas). Aquellas variables que no se- 
TABLA 1. Características basales de las dos series de pacientes

\begin{tabular}{|l|c|c|c|}
\hline & Anteroapical $(\mathrm{N}=50)$ & Anteroposterior $(\mathrm{N}=50)$ & Significación \\
\hline Edad (años) & $60 \pm 11$ & $61 \pm 12$ & $\mathrm{p}=0,51$ \\
Sexo (V/M) & $45 / 5$ & $36 / 14$ & $\mathrm{p}=0,04$ \\
Peso (kg) & $84 \pm 12$ & $83 \pm 14$ & $\mathrm{p}=0,65$ \\
FE (\%) & $58 \pm 9$ & $59 \pm 7$ & $\mathrm{p}=0,49$ \\
Tamaño AI (mm) & $43 \pm 4$ & $45 \pm 6$ & $\mathrm{p}=0,10$ \\
FC basal (lat/min) & $85 \pm 26$ & $86 \pm 23$ & $\mathrm{p}=0,86$ \\
PAM basal (mmHg) & $111 \pm 16$ & $109 \pm 17$ & $\mathrm{p}=0,56$ \\
Tiempo en FA (días) & $170 \pm 194$ & $223 \pm 554$ & $\mathrm{p}=0,57$ \\
Cardiopatía & & 4 & $\mathrm{p}=0,69$ \\
$\quad$ Isquémica & 3 & 8 & $\mathrm{p}=0,56$ \\
Valvular & 6 & 3 & $\mathrm{p}=0,01$ \\
Miocardiopatía & 13 & 19 & $\mathrm{p}=0,28$ \\
C. hipertensiva & 14 & 2 & $\mathrm{p}=0,40$ \\
Cor pulmonale & 4 & 14 & $\mathrm{p}=0,48$ \\
Ninguna & 10 & 32 & $\mathrm{p}=0,52$ \\
Antiarrítmico & & 6 & $\mathrm{p}=0,75$ \\
$\quad$ Amiodarona & 36 & 10 & $\mathrm{p}=0,79$ \\
Sotalol & 5 & 2 & $\mathrm{p}=0,55$ \\
Otros & 8 & 1 & \\
Ninguno & 1 & & \\
\hline
\end{tabular}

Valores expresados como media \pm desviación estándar. V: varón; M: mujer; AI: aurícula izquierda; FA: fibrilación auricular; FC: frecuencia cardíaca; FE: fracción de eyección; PAM: presión arterial media.

guían una distribución normal se compararon mediante U-Mann-Whitney. Se aceptó como estadísticamente significativo un valor de $\mathrm{p}<0,05$.

\section{RESULTADOS}

En el grupo A-A se trataron 50 pacientes y en el A-P otros 50, y sus características basales están recogidas en la tabla 1. Las únicas diferencias significativas encontradas fueron en el sexo, con un $10 \%$ de mujeres en el grupo A-A y un $28 \%$ en el A-P, y el porcentaje de miocardiopatía, $26 \%$ en el grupo AA frente a $6 \%$ en el A-P.

Las variables relacionadas con la cardioversión se muestran en la tabla 2. Destacamos la diferencia observada a favor de la posición A-A, que precisó menor número de choques, menor energía, y consiguió más frecuentemente la reversión al primer choque.

Sólo un paciente no revertido en la posición A-A lo fue en la A-P, mientras que 7 pacientes fallidos con electrodos A-P fueron finalmente cardiovertidos en la posición A-A.

No encontramos correlación entre la energía necesaria para la cardioversión y el peso del paciente.
Los 3 casos que presentaron deterioro hemodinámico se recuperaron sin secuelas tras administración de volumen. Todos los pacientes que precisaron ventilación con bolsa-mascarilla recuperaron respiración espontánea en pocos minutos. No hubo otros efectos secundarios y todos los pacientes fueron dados de alta a domicilio en el plazo previsto.

\section{DISCUSIÓN}

La alta prevalencia de $\mathrm{FA}^{10,11}$ y sus potenciales complicaciones ${ }^{12-14}$ justifican el gran interés de la comunidad médica en esta patología. Esto se ha traducido en la realización de estudios clínicos de impacto $^{15-17}$ y en la publicación por parte de diversas sociedades científicas de recomendaciones para el manejo de estos pacientes ${ }^{1,2,18}$. Dentro de las posibilidades terapéuticas, seleccionando adecuadamente los candidatos, la CVE constituye un tratamiento fundamental ${ }^{2,3}$, de ahí que todos los esfuerzos en aumentar su eficacia sean del máximo interés.

Diversas publicaciones hacen recomendaciones para obtener los mejores resultados con la CVE. Entre las variaciones de la técnica podemos conside-

TABLA 2. Variables relacionadas con la cardioversión

\begin{tabular}{|c|c|c|c|}
\hline & Anteroapical $(\mathrm{N}=50)$ & Anteroposterior $(\mathrm{N}=50)$ & Significación \\
\hline Reversión a RS & $48(96 \%)$ & $47(94 \%)$ & $p=0,64$ \\
\hline Número de choques* & $1(1-1)$ & $1(1-2)$ & $\mathrm{p}=0,02$ \\
\hline RS al primer choque & $37(77 \%)$ & $27(57 \%)$ & $\mathrm{p}=0,04$ \\
\hline Cambio de posición (eficaz) & 1 de $48(2 \%)$ & 7 de $47(15 \%)$ & $\mathrm{p}=0,02$ \\
\hline Energía acumulada $(\mathrm{J}) *$ & $150(150-150)$ & $150(150-300)$ & $\mathrm{p}=0,007$ \\
\hline Soporte hemodinámico & 2 & 1 & $\mathrm{p}=0,56$ \\
\hline Ventilación bolsa-mascarilla & 28 & 26 & $\mathrm{p}=0,84$ \\
\hline
\end{tabular}

El número de choques, el cambio de posición y la energía acumulada se estudian sólo en los pacientes revertidos a ritmo sinusal (RS). *Mediana (percentiles 25-75). 
rar el tipo de onda, la energía recomendada, el tipo de electrodos, la colocación de los mismos, la presión de las palas y la impedancia torácica.

Sin un consenso pleno ${ }^{19,20}$, actualmente la mayor parte de los autores encuentra superior la desfibrilación bifásica ${ }^{21-24}$, y las recomendaciones de energía inicial para este tipo de onda se sitúan entre los 100 y $200 \mathrm{~J}$ según la complexión del paciente ${ }^{21}$, pareciendo razonable utilizar $150 \mathrm{~J}$ como aproximación general.

Respecto al tipo de electrodos de desfibrilación, aunque varios autores observan menor impedancia con las palas metálicas ${ }^{4,7,25}$, hemos mantenido nuestra práctica de utilizar electrodos adhesivos por dos motivos. El primero es que permite reducir el personal encargado de la cardioversión al no precisar un operador para las palas de desfibrilación, lo que garantiza una fijación estable con posibilidad de iniciar estimulación transcutánea en caso de necesidad. El segundo es la importante reducción en la quemadura cutánea ${ }^{9,26}$

Otro aspecto interesante de la técnica es la selección de la posición de los electrodos para la cardioversión. Principalmente hay dos posiciones: $A-A$ (infraclavicular derecho -inframamilar sobre línea axilar anterior izquierda) y $A-P$ (paraesternal infraclavicular izquierdo o derecho- infraescapular izquierdo ${ }^{27}$. La mayor parte de los estudios revisados encuentran que la segunda posición es más eficaz ${ }^{7,28-}$ ${ }^{30}$, aunque ante el fallo de los choques en una posición, sugieren que se utilice la alternativa ${ }^{31}$. En los pacientes portadores de marcapasos se recomienda evitar situar los electrodos sobre el generador y parece más adecuado evitar que la dirección de la descarga coincida con la del electrocatéter, por lo que habitualmente se elige la posición A-P. Tras el procedimiento, debe comprobarse que el dispositivo funciona correctamente ${ }^{2,27}$.

En nuestra Unidad, donde son remitidos pacientes con FA crónica para CVE electiva, inicialmente se utilizaba la posición A-A como primera opción. Animados por los estudios citados decidimos modificar nuestro protocolo a la posición A-P, manteniendo el resto del tratamiento sin modificaciones. Dentro de las dos posibilidades descritas en la colocación del electrodo anterior ${ }^{27}$, elegimos colocarlo paraesternal izquierdo para conseguir una dirección de la corriente perpendicular a la posición anteroapical, y porque era la posición que utilizábamos en los pacientes portadores de marcapasos. Es reseñable la variación en la colocación de los electrodos existente entre los diferentes autores.

El resultado de nuestro estudio es que la colocación de los electrodos tanto en posición A-A como A-P era igual de efectiva para revertir a RS la FA crónica, aunque con la primera posición se necesitaba un número menor de choques y una menor energía. Somos conscientes de que se trata de un estudio observacional de dos series de pacientes, y que, por tanto, la ausencia de aleatorización constituye una importante limitación, especialmente cuando parte de la literatura revisada encuentra dis- tintos resultados ${ }^{7,28-30}$. Además, la diferencia en el porcentaje de mujeres, aun sin existir ningún estudio que relacione el sexo con el éxito en la CVE, supone una dificultad estadística que hace interpretar con cautela los resultados. También se han comprobado diferencias respecto a la cardiopatía basal (prevalencia de miocardiopatía). El número de pacientes estudiado es insuficiente para un análisis de subgrupos, pero creemos que otros aspectos caracterizan adecuadamente sus características funcionales respecto a la FA: no encontramos diferencias en el tamaño auricular izquierdo, la FE o el tiempo de evolución de la arritmia. A pesar de las limitaciones comentadas, insistimos en que nuestra casuística se basa en la aplicación del mismo protocolo, salvo la variable estudiada, a una población no sometida a ninguna selección previa (dos series consecutivas de pacientes), y que las diferencias de eficacia encontradas entre ambas posiciones de electrodos son notables.

Algunos autores explican la mayor eficacia de una determinada posición en base a una menor impedancia torácica ${ }^{30}$. Otros autores no encuentran diferencias de impedancia entre las dos posiciones, y en cambio sí que las correlacionan con los diámetros torácicos y la masa corporal ${ }^{32}$. Aunque en nuestro estudio no se midió la impedancia torácica, no encontramos relación entre el peso del paciente y la energía necesaria para conseguir RS.

Revisando brevemente la literatura sobre la posición de electrodos, podemos destacar los siguientes estudios: Mathew ${ }^{32}$ aleatoriza 90 pacientes sin encontrar diferencias en la impedancia, en el número de reversiones ni en la energía necesaria entre las dos posiciones administrando choques monofásicos; Tuinenburg $^{31}$, en su estudio con 111 pacientes también con onda monofásica observa en cambio mejores resultados con la posición anterolateral, consiguiendo incluso revertir pacientes con fallo de la posición A$\mathrm{P}$; Botto $^{28}$ encuentra mayor eficacia de la posición A-P; lo mismo que $\mathrm{Chen}^{30}$ y Kirchhof ${ }^{29}$. Este último autor ha publicado recientemente los resultados de un estudio aleatorizado con 201 pacientes, valorando el tipo de onda, el tipo de electrodos y su posicionamiento ${ }^{7}$, y observó los mejores resultados con onda bifásica a través de palas metálicas en posición A-P. Como vemos, la literatura no es concluyente, y aunque los estudios más recientes que utilizan cardioversión bifásica parecen apoyar la posición A-P, tal vez esto no sea generalizable a todas las poblaciones y justifique los resultados observados en nuestra serie. Un reciente trabajo ${ }^{33}$ que incluye 307 pacientes aleatorizados a las dos posiciones de electrodos con diferentes energías de onda bifásica, aunque observa una tendencia favorable a la posición A-A, termina concluyendo que dada la gran eficacia de los choques bifásicos, la posición de electrodos pierde su importancia.

En nuestro país la posición inicial más utilizada en la práctica es la A-A debido a que es la forma tradicional de administrar choques eléctricos, a que su aplicación es más fácil y a que es la que se enseña 
en los Cursos de Soporte Vital Avanzado ${ }^{34}$. Aunque las limitaciones de nuestro estudio impiden disipar todas las dudas al respecto, nuestros resultados (menor necesidad de energía, menos choques, más reversiones al primer choque) apoyan seguir manteniendo esta práctica. En nuestro medio parece más adecuado iniciar la cardioversión con los electrodos en posición A-A, y de no ser efectivos los choques iniciales, cambiar a la posición alternativa.

Garantizar el confort de un paciente que, según nuestro protocolo, puede recibir 5 choques antes de determinar la FA como refractaria y permitir variar la posición de los electrodos, exige que la sedación y analgesia administradas sean superiores a las utilizadas por otros grupos ${ }^{35}$. Asumiendo una mayor necesidad de ventilación con bolsa-mascarilla, hemos encontrado nuestra pauta segura, permitiendo una rápida recuperación de los pacientes con absoluta amnesia del procedimiento.

\section{Declaración de conflicto de intereses}

Los autores han declarado no tener ningún conflicto de intereses

\section{BIBLIOGRAFÍA}

1. Levy S, Breithardt G, Campbell RW, Camm AJ, Daubert JC, Allessie M, et al. Atrial fibrillation: current knowledge and recommendations for management. Working Group on Arrhythmias of the European Society of Cardiology. Eur Heart J. 1998; 19:1294-320.

2. Fuster V, Ryden LE, Asinger RW, Cannom DS, Crijns HJ, Frye RL, et al. ACC/AHA/ESC guidelines for the management of patients with atrial fibrillation: executive summary. J Am Coll Cardiol. 2001;38:1231-66.

3. Moro C, Hernández A. Cardioversión eléctrica para la fibrilación auricular. ¿Existen aún indicaciones tras el estudio AFFIRM? Rev Esp Cardiol. 2003;56:751-3.

4. Ewy GA. The optimal technique for electrical cardioversion of atrial fibrillation. Clin Cardiol. 1994;17:79-84.

5. Kerber RE. Transthoracic cardioversion of atrial fibrillation and flutter: Standard techniques and new advances. Am J Cardiol. 1996;78 Suppl 8 A:22-6.

6. Alpert MA. Medical cardioversion of atrial fibrillation. Chest. 2000;117:1529-31.

7. Kirchhof P, Monnig G, Wasmer K, Heinecke A, Breithardt G, Eckardt L, et al. A trial of self-adhesive patch electrodes and hand-held paddle electrodes for external cardioversion of atrial fibrillation (MOBIPAPA). Eur Heart J. 2005;26:1292-7.

8. Muñoz T, Castedo JF, Castañeda A, Dudagoitia JL, Poveda Y, Iribarren S. Sedación para cardioversión eléctrica. Comparación de dosis bajas de propofol y etomidato. Med Intensiva. 2002;26:98-103

9. Muñoz T, Dudagoitia JL, Poveda Y, Martínez. S, Vinuesa $\mathrm{C}$, Iribarren S, et al. Comparación de onda monofásica y bifásica en la cardioversión eléctrica de la fibrilación auricular. Med Intensiva. 2005;29:79-82

10. Ostranderld JR, Brandt RL, Kjelsberg MO, Epstein FH. Electrocardiographic findings among the adult population of a total natural community, Tecumseh, Michigan. Circulation. 1965; 31:888-98.

11. Furberg CD, Psaty BM, Manolio TA, Gardin JM, Smith VE, Rautaharju PM. Prevalence of atrial fibrillation in elderly subjets (the Cardiovascular Health Study). Am J Cardiol. 1994; $74: 236-41$
12. Wolf PA, Abbott RD, Kannel WB. Atrial fibrillation as an independent risk factor for stroke: the Framingham Study. Stroke. 1991:22:983-8.

13. Krahn AD, Manfreda J, Tate RB, Mathweson FA, Cuddy TE. The natural history of atrial fibrillation: incidence, risk factors and prognosis in the Manitoba Follow-up Study. Am J Med. 1995; $98: 476-84$

14. Chugh SS, Blackster JL, Shen WK, Hammill SC, Gersh BJ. Epidemiology and Natural History of Atrial Fibrillation: Clinical Implications. J Am Coll Cardiol. 2001;37:371-8.

15. Hohnloser SH, Kuck KH, Lillenthal J. Rhythm or rate control in atrial fibrillation - Pharmacological Intervention in Atrial Fibrillation (PIAF): a randomised trial. Lancet. 2000;356: 1789-94.

16. Wyse DG, Waldo AL, DiMarco JP, Domanski MJ, Rosenberg Y, Schron EB, et al. A comparison of rate control and rhythm control in patients with atrial fibrillation. $\mathrm{N}$ Eng $\mathrm{J}$ Med. 2002;347:1825-33.

17. Hagens VE, Vermeulen KM, TenVergert EM, Van Veldhuisen DJ, Bosker HA, Kamp O, et al. Rate control is more cost-effective than rhythm control for patients with persistent atrial fibrillation - results from the RAte Control versus Electrical cardioversion (RACE) study. Eur Heart J. 2004;25:1542-9.

18. Khand AU, Rankin AC, Kaye GC, Cleland JG. Systematic Review of the management of atrial fibrillation in patients with heart failure. Eur Heart J. 2000;21:614-32.

19. Morgan JM. Importance of using biphasic shock waveforms for cardioversion from atrial fibrillation: an unresolved issue. Heart. 2004;90:1105-6.

20. Kim ML, Kim SG, Park DS, Gross JN, Ferrick KJ, Palma EC, et al. Comparison of rectilinear biphasic waveform energy versus truncated exponential biphasic waveform energy for transthoracic cardioversion of atrial fibrillation. Am J Cardiol. 2004:94:1438-40.

21. Mittal S, Ayati S, Stein KM, Schwartzman D, Cavlovich D, Tchou PJ, et al. Transthoracic cardioversion of atrial fibrillation. comparison of rectilinear biphasic versus damped sine wave monophasic shocks. Circulation. 2000;101:1282-7.

22. Page RL, Kerber RE, Russell JK, Trouton T, Waktare J, Gallik D, et al. Biphasic versus monophasic shock waveform for conversion of atrial fibrillation. J Am Coll Cardiol. 2002;39: 1956-63.

23. Khaykin $\mathrm{Y}$, Newman D, Kowalewski M, Korley V, Dorian P. Biphasic versus monophasic cardioversion in shock-resistant atrial fibrillation. J Cardiovasc Electrophysiol. 2003;14: 868-72.

24. Scholten M, Szili-Torok T, Klootwijk P, Jordaens L. Comparison of monophasic and biphasic shocks for transthoracic cardioversion of atrial fibrillation. Heart. 2003;89:1032-4.

25. Dodd TE, Deakin CD, Petley GW, Clewlow F. External defibrillation in the left lateral position: a comparison of manual paddles with self-adhesive pads. Resuscitation. 2004:63:283-6.

26. Ambler JJS, Sado DM, Zideman DA, Deakin CD. The incidence and severity of cutaneous burns following external DC cardioversion. Resuscitation. 2004;61:281-8

27. Schoonderwoerd BA, Van Gelder IC, Crijns HJGM. External electrical cardioversión of atrial fibrillation. En: Brugada J, editor. Atrial fbrillation, a practical approach. Barcelona: Prous Science; 2000. p. 95-102.

28. Botto GL, Politi A, Bonini W, Broffoni T, Bonatti R. External cardioversion of atrial fibrillation: role of paddle position on technical efficacy and energy requirements. Heart. 1999; 82:726-30.

29. Kirchhof P, Borggrefe M, Breithardt G. Effect of electrode position on the outcome of cardioversion. Card Electrophysiol Rev. 2003;7:292-6.

30. Chen CJ, Guo GB. External cardioversion in patients with persistent atrial fibrillation: a reappraisal of the effects of electrode pad position and transthoracic impedance on cardioversion success. Jpn Heart J. 2003;44:921-32.

31. Tuinenburg AE, Schoonderwoerd BA, Van Gelder IC. The sequential application of two different paddle positions in external atrial defibrillation. Eur Heart J. 1999,20: 592.

32. Mathew TP, Moore A, McIntyre M, Harbinson MT, Campbell NP, Adgey AA, et al. Randomised comparison of elec- 
trode positions for cardioversion of atrial fibrillation. Heart. 1999; 81:576-9.

33. Walsh SJ, McCarty D, McClelland AJ, Owens CG, Trouton TG, Harbinson MT, et al. Impedance compensated biphasic waveforms for transthoracic cardioversion of atrial fibrillation: a multi-centre comparison of antero-apical and antero-posterior pad positions. Eur Heart J. 2005;26:1298-302
34. Tormo C, Ruano M, Bonastre J. Tratamiento eléctrico de las arritmias. En: Ruano M, Tormo C, editores. Manual de Soporte Vital Avanzado. 3. ${ }^{\mathrm{a}}$ ed. Barcelona: Masson SA; 2004. p. 12138.

35. Muñoz T, Castedo JF, Dudagoitia JL, Poveda Y, Iribarren $\mathrm{S}$, Castañeda A, et al. Cardioversión eléctrica bajo anestesia con bolus único de propofol y remifentanilo. Forhos. 2002;5:7-11. 